\title{
A COMBINATORIAL FORMULA WITH SOME APPLICATIONS
}

\section{CHING-SIUR HSU}

The aim of this note is to present a combinatorial formula and state its applications to partitions, number of solutions, and Dirichlet's integral.

Let $\theta_{1}(x), \cdots, \theta_{n}(x)$ be $n$ arbitrary functions of $x$ and let ${ }_{m} \Im_{a_{1} \ldots a_{n}}^{b_{1} \ldots b_{n}}\left(\left[\theta_{1}\right] \cdots\left[\theta_{n}\right]\right)$ be defined by

$$
\begin{gathered}
{ }_{m} \Im_{a_{1} \cdots a_{n}}^{b_{1} \cdots b_{n}}\left(\left[\theta_{1}\right] \cdots\left[\theta_{n}\right]\right) \\
=\sum_{x_{1}+\cdots+\dot{x}_{n}=m, a_{1} \leqq x_{1} \leqq b_{1} \cdots a_{n} \leqq b_{n}} \theta_{1}\left(x_{1}\right) \cdots \theta_{n}\left(x_{n}\right),
\end{gathered}
$$

where $a_{1}, \cdots, a_{n}, b_{1}, \cdots, b_{n}, m$ are all integers and the right-hand side of (1) is summed over all different integral solutions of $x_{1}+\cdots+x_{n}=m$ with $a_{1} \leqq x_{1} \leqq b_{1}, \cdots, a_{n} \leqq x_{n} \leqq b_{n}$.

More generally we define

$$
\begin{aligned}
& { }_{m} S_{a_{1} \cdots a_{n}}^{b_{1} \cdots b_{n}}\left(\left[\theta_{1}\right]^{n_{1}} \cdots\left[\theta_{k}\right]^{n_{k}}\right) \\
& \quad \sum_{x_{11}+\cdots+x_{n_{1}}+\cdots+x_{k 1}+\cdots+x_{k n_{k}}=m, a_{1} \leqq x_{1} \leqq b_{1}, \cdots, a_{k} \leqq x_{k i} \leqq b_{k}} \theta_{1}\left(x_{11}\right) \\
& \cdots \theta_{1}\left(x_{1 n_{1}}\right) \cdots \theta_{k}\left(x_{k 1}\right) \cdots \theta_{k}\left(x_{k n_{k}}\right) .
\end{aligned}
$$

We make the following conventions: ${ }^{1}$

(A) ${ }_{m} \Im_{a}^{b}\left([\theta]^{n}\right)=0$ for $m<n a$ or $m>n b$.

(B) ${ }_{m} \subseteq\left([\theta]^{0}\right)=0$ if $m \neq 0,{ }_{m} \subseteq\left([\theta]^{0}\right)=1$ if $m=0$.

(C) If $a_{1}=\cdots=a_{n}=a, b_{1}=\cdots=b_{n}=b$, we write

$$
{ }_{m} \Im_{a_{1} \cdots a_{n}}^{b_{1} \cdots b_{n}}\left(\left[\theta_{1}\right] \cdots\left[\theta_{n}\right]\right) \text { as }{ }_{m} \Im_{a}^{b}\left(\left[\theta_{1}\right] \cdots\left[\theta_{n}\right]\right) .
$$

We now show that ${ }^{2}$

$$
\begin{aligned}
& \sum_{\nu_{1}=0}^{n_{1}} \sum_{\nu_{2}=0}^{n_{2}} \cdots \sum_{\nu_{k}=0}^{n_{k}}(-1)^{\nu_{1}+\nu_{2}+\cdots+\nu_{k}} C_{n_{1}, \nu_{1}} C_{n_{2}, \nu_{2}} C_{n_{k}, \nu_{k}} \\
& \begin{array}{r}
\cdot{ }_{m^{\prime}} \Im_{1}^{\infty}\left(\left[\phi_{1}\right]^{\nu_{1}}\left[\psi_{1}\right]^{n_{1}-\nu_{1}} \cdots\left[\phi_{k}\right]^{\nu_{k}}\left[\psi_{k}\right]^{n_{k}-\nu_{k}}\right) \\
={ }_{m} \Im_{a_{1} \cdots a_{k}}^{b_{1} \cdots b_{k}}\left(\left[\theta_{1}\right]^{n_{1}} \cdots\left[\theta_{k}\right]^{n_{k}}\right),
\end{array}
\end{aligned}
$$

where $\theta_{1}, \cdots, \theta_{k}$ are $k$ arbitrary functions of $x$ and $\phi_{i}(x)=\theta_{i}\left(x+b_{i}\right)$,

Received by the editors June 2, 1944 .

1 These conventions are used in proving (3) and other formulas.

2 The formula (3) shows that all the strong restrictions for $x$ can be removed. 
$\psi_{i}(x)=\theta_{i}\left(x+a_{i}-1\right), m^{\prime}=m^{\prime}\left(\nu_{1} \cdots \nu_{k}\right)=m-\sum b_{i} \nu_{i}-\sum\left(a_{i}-1\right)\left(n_{i}-\nu_{i}\right)$ $(i=1, \cdots, k)$.

ProOF. By definition we have

$$
\begin{aligned}
& m^{\prime} \Im_{1}^{\infty}\left(\left[\phi_{1}\right]^{\nu_{1}}\left[\psi_{1}\right]^{n_{1}-\nu_{1}} \cdots\right. {\left.\left[\phi_{k}\right]^{\nu_{k}}\left[\psi_{k}\right]^{n_{k-\nu_{k}}}\right) } \\
&= \sum_{m_{1}+\cdots+m_{2 k}=m}{ }_{m_{1}} \Im_{b_{1}+1}^{\infty}\left(\left[\theta_{1}\right]^{\nu_{1}}\right)_{m_{2}} \Im_{a_{1}}^{\infty}\left(\left[\theta_{1}\right]^{n_{1}-\nu_{1}}\right) \\
& \cdots{ }_{m_{2 k}-1} \Im_{b_{k+1}}^{\infty}\left(\left[\theta_{k}\right]^{\nu_{k}}\right)_{m_{2 k}} \Im_{a_{k}}^{\infty}\left(\left[\theta_{k}\right]^{n_{k}-\nu_{k}}\right) .
\end{aligned}
$$

Let $T_{i}=\theta_{i}\left(x_{i 1}\right) \cdots \theta_{i}\left(x_{i n_{i}}\right)(i=1, \cdots, k)$, and let $T=T_{1} \cdots T_{k}$ be a term contained in the right-hand side of (3), that is, $x_{1} \geqq a_{1}, \cdots, x_{k}$ $\geqq a_{k}$. Without loss of generality we may assume $x_{11}, \cdots, x_{1 t_{1}}$ $\geqq b_{1}+1 ; \cdots ; x_{k 1}, \cdots, x_{k t_{k}} \geqq b_{k}+1$. Since the necessary and sufficient condition for

$$
T_{i} \in{ }_{m_{2 i-1}} \Im_{b_{i}+1}^{\infty}\left(\left[\theta_{i}\right]^{\nu_{i}}\right)_{m_{2 i}} \Im_{a_{i}}^{\infty}\left(\left[\theta_{i}\right]^{n_{i}-v_{i}}\right) \quad(1 \leqq i \leqq k)
$$

is that there is a term $\theta_{i}\left(x_{1}\right) \cdots \theta_{i}\left(x_{v_{i}}\right)$ of ${ }_{m_{2 i-1}} \mathfrak{S}_{b_{i+1}}^{\infty}$ contained in $T_{i}$ as a part while the other part $T_{i} / \theta_{i}\left(x_{1}\right) \cdots \theta_{i}\left(x_{\nu_{i}}\right)$ is contained in ${ }_{m_{2 i}} \Im_{a_{i}}^{\infty}$, the number of occurrences of $T$ in the left-hand side of (3) is therefore given by

$$
\begin{array}{r}
\left\{\sum_{\nu_{1}=0}^{t_{1}}(-1)^{\nu_{1}} C_{t_{1}, \nu 1}\right\}\left\{\sum_{\nu_{2}=0}^{t_{2}}(-1)^{\nu_{2}} C_{t_{2}, \nu_{2}}\right\} \\
= \begin{cases}0 & \text { if } t_{1}, \cdots, t_{k}=0 \text { are not all zero, } \\
1 & \text { if } t_{1}=\cdots=t_{k}=0 .\end{cases}
\end{array}
$$

We see that the term $T$ generally vanishes except when $a_{j} \leqq x_{j i} \leqq b_{j}$ $(j=1, \cdots, k)$. Hence ( 3$)$ is proved.

It is directly deduced from (3) by putting $n_{1}=\cdots=n_{k}=1$, $n_{1}+\cdots+n_{k}=n$ that

$$
\begin{aligned}
\sum_{k=0}^{n}(-1)^{k} \sum_{\left(\alpha_{1} \ldots \alpha_{k}\right) \in(1 \cdots n)}{ }_{m^{\prime}} \Im_{1}^{\infty}\left(\left[\phi_{\alpha_{1}}\right]\right. & \left.\cdots\left[\phi_{\alpha_{k}}\right]\left[\psi_{\alpha_{k+1}}\right] \cdots\left[\psi_{\alpha_{n}}\right]\right) \\
& ={ }_{m} \Im_{a_{1}}^{b_{1} \cdots b_{n}}\left(\left[\theta_{1}\right] \cdots\left[\theta_{n}\right]\right),
\end{aligned}
$$

where $\left(\alpha_{1} \cdots \alpha_{k} \cdots \alpha_{n}\right)=(1 \cdots n), m^{\prime}=m^{\prime}\left(\alpha_{1} \cdots \alpha_{k}\right)=m+n-k$ $-\left(b_{\alpha_{1}}+\cdots+b_{\alpha_{k}}+a_{\alpha_{k+1}}+\cdots+a_{\alpha_{n}}\right)$.

Let $F$ be an arbitrary function of $\theta_{1}, \cdots, \theta_{n}$ and let ${ }_{m} \Im_{a_{1} \cdots a_{n}}^{b_{1} \ldots b_{n}}\left\{F\left(\theta_{1}, \cdots, \theta_{n}\right)\right\}$ be defined by

$$
\begin{aligned}
{ }_{m} \Im_{a_{1} \cdots a_{n}}^{b_{1} \cdots b_{n}}\left\{F\left(\theta_{1}, \cdots, \theta_{n}\right)\right\} & \sum_{x_{1}+\cdots+x_{n}=m, a_{1} \leqq x_{1} \leqq b_{1}, \cdots, a_{n} \leqq x_{n} \leqq b_{n}} F\left(\theta_{1}\left(x_{1}\right), \cdots, \theta_{n}\left(x_{n}\right)\right) .
\end{aligned}
$$


Then, the formula (4) can be written more generally as

$$
\begin{array}{r}
\sum_{k=0}^{n}(-1)^{n} \sum_{\left(\alpha_{1} \ldots \alpha_{k}\right) \in(1 \cdots n)}{ }_{m^{\prime}} \mathbb{S}_{1}^{\infty}\left\{F\left(\phi_{\alpha_{1}}, \cdots, \phi_{\alpha_{k}}, \psi_{\alpha_{k+1}}, \cdots, \psi_{\alpha_{n}}\right)\right\} \\
={ }_{m} \Im_{a_{1} \cdots a_{n}}^{b_{1} \cdots b_{n}}\left\{F\left(\theta_{1}, \cdots, \theta_{n}\right)\right\} .
\end{array}
$$

If, for every $\left(\alpha_{1} \cdots \alpha_{k}\right)$, the limit

$$
\lim _{m \rightarrow \infty}{ }_{m^{\prime}} \mathfrak{S}_{1}^{\infty}\left\{F\left(\phi_{\alpha_{1}}, \cdots, \phi_{\alpha_{k}}, \psi_{\alpha_{k+1}}, \cdots, \psi_{\alpha_{n}}\right)\right\} \quad\left(m^{\prime}=m^{\prime}\left(\alpha_{1} \cdots \alpha_{k}\right)\right)
$$

exists, then we have further

$$
\begin{aligned}
& \sum_{(6)^{k=0}}^{n}(-1)^{k} \sum_{\left(\alpha_{1} \cdots \alpha_{k}\right) \in(1, \ldots n)} \lim _{m \rightarrow \infty} m^{\prime} \mathbb{S}_{1}^{\infty}\left\{F\left(\phi_{\alpha_{1}}, \cdots, \phi_{\alpha_{k}}, \psi_{\alpha_{k+1}}, \cdots, \psi_{\alpha_{n}}\right)\right\} \\
&=\lim _{m \rightarrow \infty}{ }_{m} \mathfrak{S}_{a_{1} \cdots b_{n}}^{b_{1} \cdots b_{n}}\left\{F\left(\theta_{1}, \cdots, \theta_{n}\right)\right\} .
\end{aligned}
$$

We shall now state some applications of the above formulas.

Application to partitions. We denote by $p_{n}(m)$ the number of partitions of $m$ into parts not exceeding $n$ or into at most $n$ parts. Let $\left\{\beta_{1} \cdots \beta_{n}\right\}$ be an ordered partition of $m$, namely,

$$
m=\beta_{1}+\beta_{2}+\cdots+\beta_{n}, \quad \beta_{1} \geqq \beta_{2} \geqq \cdots \geqq \beta_{n} .
$$

We further denote by $p_{a_{1} \ldots a_{n}}^{b_{1} \ldots b_{n}}(m)$ the number of ordered partitions, $\left\{\beta_{1} \cdots \beta_{n}\right\}$ 's, of $m$ into exactly $n$ parts which are restricted to $a_{1} \leqq \beta_{1} \leqq b_{1}, a_{2} \leqq \beta_{2} \leqq b_{2}, \cdots, a_{n} \leqq \beta_{n} \leqq b_{n}$. We have then

$$
\text { (7) } \begin{array}{r}
p_{a_{1} \cdots a_{n}}^{b_{1} \cdots b_{n}}(m)=\sum_{k=0}^{n}(-1)^{k} \sum_{\left(\alpha_{1} \cdots \alpha_{k}\right) \in(1 \cdots n)} p_{n}\left(m-k-b_{\alpha_{1}}-\cdots\right. \\
\left.-b_{\alpha_{k}}-a_{\alpha_{k+1}}-\cdots-a_{\alpha_{n}}\right),
\end{array}
$$

where the $p_{n}(m)$ 's can be evaluated by the generating function

(8) $\quad G_{n}(x)=\frac{1}{(1-x)\left(1-x^{2}\right) \cdots\left(1-x^{n}\right)}=1+\sum_{m=1}^{\infty} p_{n}(m) x^{m}$.

Proof of (7). Starting with (5) we define

$$
\theta_{i}(x)= \begin{cases}1 & \text { if } \quad a_{i} \leqq x \leqq b_{i}, \\ 0 & \text { if } \quad x<a_{i} \text { or } b_{i}<x, \quad(i=1 \cdots n),\end{cases}
$$

$F\left(\theta_{1}\left(x_{1}\right), \cdots, \theta_{n}\left(x_{n}\right)\right)$

$= \begin{cases}\theta_{1}\left(x_{1}\right) \cdots \theta_{n}\left(x_{n}\right) & \text { if the order } x_{1} \geqq x_{2} \geqq \cdots \geqq x_{n} \text { holds, } \\ 0 & \text { if the order } x_{1} \geqq x_{2} \geqq \cdots \geqq x_{n} \text { does not hold. }\end{cases}$ 
Thus we see that

Similarly

$$
{ }_{m} \Im_{a_{1} \cdots a_{n}}^{b_{1} \cdots b_{n}}\left\{F\left(\theta_{1}, \cdots, \theta_{n}\right)\right\}=p_{a_{1} \cdots a_{n}}^{b_{1} \cdots b_{n}}(m) .
$$

$$
\begin{aligned}
{ }_{m^{\prime}} \Im_{1}^{\infty}\left\{F\left(\phi_{\alpha_{1}}, \cdots, \phi_{\alpha_{k}}, \psi_{c_{k+1}}, \cdots, \psi_{\alpha_{n}}\right)\right\} \\
\quad=p_{n}\left(m-b_{\alpha_{1}}-\cdots-b_{\alpha_{k}}-a_{\alpha_{k+1}}-\cdots-a_{\alpha_{n}}-k\right) .
\end{aligned}
$$

Hence (7) is proved.

It may be noted that the formula (7) still holds when $p_{a_{1} \ldots a_{n}}^{b_{1} \ldots b_{n}}(m)$ denotes the number of partitions of $m$ into $n$ parts which are restricted to more conditions than

$$
\beta_{1} \geqq \beta_{2} \geqq \cdots \geqq \beta_{n} ; \quad a_{1} \leqq \beta_{1} \leqq b_{1}, \cdots, a_{n} \leqq \beta_{n} \leqq b_{n} .
$$

Application to number of solutions. Let $A_{a_{1} \ldots a_{n}}^{b_{1} \ldots b_{n}}(N)$ denote the number of integral (or prime) solutions of the equation

$$
x_{1}^{k}+x_{2}^{k}+\cdots+x_{n}^{k}=N
$$

with $a_{1} \leqq x_{1}<b_{1}, a_{2} \leqq x_{2}<b_{2}, \cdots, a_{n} \leqq x_{n}<b_{n}$, then by (5) we have

$$
\text { (9) } A_{a_{1} \cdots a_{n}}^{b_{1} \cdots b_{n}}(N)=\sum_{k=0}^{n}(-1)^{k} \sum_{\left(\nu_{1} \cdots \nu_{k}\right) \in(1 \cdots n)} A_{b_{\nu_{1}} \cdots b_{\nu_{k}} a_{\nu_{k+1}} \cdots a_{\nu_{n}}}^{\infty}(N) \text {, }
$$

where $\left(\nu_{1} \cdots \nu_{k} \cdots \nu_{n}\right)=(1 \cdots n)$.

We shall now proceed to find an asymptotic formula concerning the number of integral solutions of a linear equation with integral coefficients.

Let $A(N)$ denote the number of integral solutions of the equation

$$
\sum_{k=1}^{n} c_{k} x_{k}=N \quad\left(c_{1} \geqq 1, \cdots, c_{n} \geqq 1\right)
$$

under the restrictions $\alpha_{k} N<x_{k}<\beta_{k} N(k=1, \cdots, n)$, where $c_{1}, \cdots, c_{n}$ are relatively prime to each other, $\alpha_{k}, \beta_{k}$ are real values.

When $\alpha_{k}=0, \beta_{k}=1(k=1, \cdots, n)$ it is well known that ${ }^{3}$

$$
A(N)=N^{n-1} / c_{1} \cdots c_{n} \cdot(n-1) !+O\left(N^{n-2}\right) .
$$

Define

$\theta_{i}(x)=\left\{\begin{array}{l}1 \text { if } x \text { is divisible by } c_{i} \text { and the inequality } \alpha_{i} c_{i} N<x<\beta_{i} c_{i} N \text { holds, } \\ 0 \text { if } x \text { is not divisible by } c_{i} \text { or the inequality does not hold. }\end{array}\right.$

${ }^{3}$ The proof of (10) can be obtained easily by the method of partial fractions. 
Thus by (4) and (10) we obtain the following consequence.4

Let $S_{k}=\sum_{\left(\nu_{1} \ldots \nu_{k}\right) \in(1 \cdots n)} \phi_{\nu_{1}} \ldots \nu_{k}$, where

$\phi_{\nu_{1}} \ldots \nu_{k}=\left\{\begin{array}{rr}0 & \text { for }\left(1-\beta_{\nu_{1}} c_{\nu_{1}}-\cdots-\alpha_{v_{n}} c_{\nu_{n}}\right) \leqq 0, \\ \left(1-\beta_{\nu_{1}} c_{\nu_{1}}-\cdots-\beta_{\nu_{k}} c_{\nu_{k}}-\alpha_{\nu_{k+1}} c_{\nu_{k+1}}-\cdots-\alpha_{\nu_{n}} c_{\nu_{n}}\right)^{n-1} & \text { for }\left(1-\beta_{\nu_{1}} c_{\nu_{1}}-\cdots-\alpha_{\nu_{n}} c_{\nu_{n}}\right)>0 .\end{array}\right.$

Then

$$
\frac{A(N)}{N^{n-1}}=\frac{S_{0}-S_{1}+\cdots \pm S_{n}}{c_{1} \cdots c_{n} \cdot(n-1) !}+O\left(\frac{1}{N}\right) .
$$

Evidently (11) may be seen as a generalization of (10). If $c_{1}=\cdots=c_{n}=1, a_{k} \leqq x_{k} \leqq b_{k}(k=1, \cdots, n)$, we can express $A(N)$ more precisely as $^{5}$

(12) $A(N)=\sum_{k=0}^{n}(-1)^{k} \sum_{\left(\nu_{1} \cdots \nu_{k}\right) \in(1 \cdots n)} C_{N^{\prime}(k)+\left(a_{\nu_{1}}+\cdots+a_{\nu_{k}}\right)-\left(b_{\nu_{1}}+\cdots+b_{\nu_{k}}\right), n-1}$ where $N^{\prime}(k)=N+n-k-1-\left(a_{1}+\cdots+a_{n}\right)$.

Application to Dirichlet's integral. The following theorem is well known.

Let

$$
\begin{aligned}
& I_{(0)}=\iint_{D} \cdots \int x_{1}^{\alpha_{1}-1} x_{2}^{\alpha_{2}-1} \cdots x_{n}^{\alpha_{n}-1} \\
& \cdot f\left(x_{1}+x_{2}+\cdots+x_{n}\right) d x_{1} d x_{2} \cdots d x_{n} \text {, }
\end{aligned}
$$

where the variables $x_{1}, x_{2}, \cdots, x_{n}$ are restricted to the region

$D: 0 \leqq k_{1} \leqq x_{1}+x_{2}+\cdots+x_{n} \leqq k_{2} ; 0 \leqq x_{1}, 0 \leqq x_{2}, \cdots, 0 \leqq x_{n}$.

Then the integral $I_{(0)}$ can be reduced to the form

$$
I_{(0)}=\frac{\Gamma\left(\alpha_{1}\right) \Gamma\left(\alpha_{2}\right) \cdots \Gamma\left(\alpha_{n}\right)}{\Gamma\left(\alpha_{1}+\alpha_{2}+\cdots+\alpha_{n}\right)} \int_{k_{1}}^{k_{2}} u^{\alpha_{1}+\alpha_{2}+\cdots+\alpha_{n}-1} f(u) d u .
$$

We shall now extend the formula (13). Let

$$
\begin{aligned}
& I\left\{\begin{array}{l}
b_{1}, \cdots, b_{n} \\
x_{1}, \cdots, x_{n} \\
a_{1}, \cdots, a_{n}
\end{array}\right\}=\iint_{R} \cdots \int x_{1}^{\alpha_{1}-1} x_{2}^{\alpha_{2}-1} \cdots x_{n}^{\alpha_{n}-1} \\
& \cdot f\left(x_{1}+x_{2}+\cdots+x_{n}\right) d x_{1} d x_{2} \cdots d x_{n},
\end{aligned}
$$

${ }^{4}$ The detailed proof of this is omitted.

${ }^{5}$ The formula (12) can be obtained also by considering two generating functions. 
where the region $R$ is defined as the set of all points $\left(x_{1} \cdots x_{n}\right)$ such that

$$
0 \leqq a_{i} \leqq x_{i} \leqq b_{i} ; \quad x_{1}+x_{2}+\cdots+x_{n} \leqq k .
$$

Since the Dirichlet integral $I_{(0)}$ can be considered as a limit of multiple summations with variable upper limits, by applying (6) we have

$$
\begin{aligned}
& I\left\{\begin{array}{l}
b_{1} \cdots b_{n} \\
x_{1} \cdots x_{n} \\
a_{1} \cdots a_{n}
\end{array}\right\} \\
& =\sum_{j=0}^{n}(-1)^{j} \sum_{\left(\nu_{1} \ldots v_{j}\right) \in(1 \cdots n)} I\left\{\begin{array}{llllll}
\infty & \cdots & \infty & \infty & \cdots & \infty \\
x_{\nu_{1}} & \cdots & x_{\nu_{j}} & x_{\nu_{j+1}} & \cdots & x_{v_{n}} \\
b_{v_{1}} & \cdots & b_{\nu_{j}} & a_{\nu_{j+1}} & \cdots & a_{\nu_{n}}
\end{array}\right\} .
\end{aligned}
$$

We have to establish a formula for

$$
I\left\{\begin{array}{l}
\infty \cdots \infty \\
x_{1} \cdots x_{n} \\
c_{1} \cdots c_{n}
\end{array}\right\} \quad\left(c_{1} \geqq 0, \cdots, c_{n} \geqq 0\right) .
$$

Let $I_{(1 \ldots s)}$ denote the integral

$$
\begin{gathered}
\frac{\Gamma\left(\alpha_{s+1}\right) \cdots \Gamma\left(\alpha_{n}\right)}{\Gamma\left(\alpha_{s+1}+\cdots+\alpha_{n}\right)} \int_{0}^{\min \left(c_{1}, k\right)} x_{1}^{\alpha_{1}-1} d x_{1} \int_{0}^{\min \left(c_{2}, k-x_{1}\right)} x_{2}^{\alpha_{2}-1} d x_{2} \\
\cdots \int_{0}^{\min \left(c_{s}, k-x_{1}-\cdots-x_{s-1}\right)} x_{s}^{\alpha_{s}-1} d x_{s} \\
\quad \int_{0}^{k-x_{1}-\cdots-x_{s}} u^{\alpha_{s+1}+\cdots+\alpha_{n}-1} f\left(u+x_{1}+\cdots+x_{s}\right) d u .
\end{gathered}
$$

Now, by (14) it can be shown that

$$
\begin{gathered}
I\left\{\begin{array}{l}
\infty \cdots \infty \\
x_{1} \cdots x_{n} \\
c_{1} \cdots c_{n}
\end{array}\right\}=I_{(0)}-\sum_{(i) \in(1 \cdots n)}^{c_{n, 1}} I_{(i)}+\sum_{(i j) \in(1 \cdots n)}^{c_{n, 2}} I_{(i j)}-\cdots \\
+(-1)^{n} I_{(1 \cdots n)}
\end{gathered}
$$

where

$$
\begin{aligned}
& I_{(0)}=I\left\{\begin{array}{lll}
\infty & \cdots & \infty \\
x_{1} & \cdots & x_{n} \\
0 & \cdots & 0
\end{array}\right\} \\
& I_{(1 \cdots n)}=\int_{0}^{\min \left(c_{1}, k\right)} \cdots \int_{0}^{\min \left(c_{n}, k-x_{1}-\cdots-x_{n-1}\right)} x_{1}^{\alpha_{1-1}} \cdots x_{n}^{\alpha_{n-1}} d x_{1} \cdots d x_{n} .
\end{aligned}
$$


To prove (15), the formal logic theorem is also applicable.

Consider a differential $\bar{x}_{1}^{\alpha_{1}-1} \cdots \bar{x}_{n}^{\alpha_{n}-1} f\left(\bar{x}_{1}+\cdots+\bar{x}_{n}\right) d \bar{x}_{1} \cdots d \bar{x}_{n}$. We may assume that $\bar{x}_{v_{1}} \leqq c_{v_{1}}, \cdots, \bar{x}_{v_{t}} \leqq c_{v_{t}}, \bar{x}_{v_{t+1}}>c_{v_{t+1}}, \cdots, \bar{x}_{v_{n}}>c_{v_{n}}$, $\left(\nu_{1} \cdots \nu_{n}\right)=(1 \cdots n)$.

Since the integral is a limit of multiple summations and may be written as

$$
\begin{aligned}
& I_{(1 \cdots s)}= \int_{0}^{\min \left(c_{1}, k\right)} x_{1}^{\alpha_{1-1}} d x_{1} \cdots \int_{0}^{\min \left(c_{s}, k-x_{1}-\cdots-x_{s-1}\right)} x_{s}^{\alpha_{s}-1} d x_{s} \\
& \cdot \int_{R_{1}} \cdots \iint_{x_{s+1}^{\alpha_{s+1}-1}} \cdots x_{n}^{\alpha_{n-1}} f\left(x_{1}+\cdots+x_{n}\right) d x_{s+1} \cdots d x_{n} \\
&= \int_{0}^{c_{1}} \cdots \int_{0}^{c_{s}} \int_{R_{2}\left(x_{1} \cdots x_{n}\right)} \cdots \int x_{1}^{\alpha_{1-1}} \cdots x_{s}^{\alpha_{s}-1} x_{s+1}^{\alpha_{s+1}} \cdots x_{n}^{\alpha_{n}} \\
& \cdot f\left(x_{1}+\cdots+x_{n}\right) d x_{1} \cdots d x_{n}
\end{aligned}
$$

where

$$
\begin{array}{lll}
R_{1}\left(x_{s+1} \cdots x_{n}\right): & 0 \leqq x_{s+1}+\cdots+x_{n} \leqq k-\left(x_{1}+\cdots+x_{s}\right) ; \\
R_{2}\left(x_{1} \cdots x_{n}\right): & 0 \leqq x_{1} \leqq c_{1}, \cdots, 0 \leqq x_{s} \leqq c_{s} ; & \\
& 0 \leqq x_{1}+\cdots+x_{n} \leqq k ; & x_{s+1}, \cdots, x_{n} \leqq 0,
\end{array}
$$

we see that the differential $\bar{x}_{1}^{\alpha_{1}-1} \cdots \bar{x}_{n}^{\alpha-1} f\left(\bar{x}_{1}+\cdots+\bar{x}_{n}\right) d \bar{x}_{1} \cdots d \bar{x}_{n}$ appears exactly $C_{t, s}$ times in $\sum_{\left(\nu_{1} \ldots \nu_{s}\right) \in(1 \cdots n)} I_{\left(\nu_{1} \ldots \nu_{s}\right)}$. Therefore the number of occurrences of the given differential in the right-hand side of (15) is equal to

$$
C_{t, 0}-C_{t, 1}+\cdots+(-1)^{t} C_{t, t}=\left\{\begin{array}{lll}
1 & \text { if } t=0 \\
0 & \text { if } t>0
\end{array}\right.
$$

Hence the formula (15) is proved.

The integral $I_{(1 \ldots s)}$ may be calculated by dividing the limits of the integral and integrating it separately.

It is seen that the integral $I_{(1} \ldots$ s) can be written also in the form:

$$
\begin{aligned}
& \frac{\Gamma\left(\alpha_{s+1}\right) \cdots \Gamma\left(\alpha_{n}\right)}{\Gamma\left(\alpha_{s+1}+\cdots+\alpha_{n}\right)} \int_{0}^{c_{1}} x_{1}^{\alpha_{1}-1} d x_{1} \int_{0}^{c_{2}} x_{2}^{\alpha_{2}-1} d x_{2} \cdots \int_{0}^{c_{s}} x_{s}^{\alpha_{s}-1} d x_{s} \\
& \cdot \int_{0}^{\left.\left[\left(k-x_{1}-\cdots-x_{s}\right)+\mid k-x_{1}-\cdots-x_{s}\right]\right] / 2} u^{\alpha_{s+1}+\cdots+\alpha_{n}-1} f\left(u+x_{1}+\cdots+x_{s}\right) d u .
\end{aligned}
$$

Connecting (14) with (15), we see that it is a generalization of Dirichlet's integral $I_{(0)}$ with $k_{2}=0, k_{1}=k$ in (13). 
It may be noted that the formula (14) is also called Liouville's extension and the integral regions $D$ and $R$ can be defined also by

$$
\begin{aligned}
& D: \quad 0 \leqq k_{1} \leqq a_{1} x_{1}^{p_{1}}+\cdots+a_{n} x^{p_{n}} \leqq k_{2}, \quad 0 \leqq x_{i} ; \\
& R: \quad 0 \leqq a_{i} \leqq x_{i} \leqq b_{i}, \quad d_{1} x_{1}^{q_{1}}+\cdots+d x_{n}^{q_{n}} \leqq k .
\end{aligned}
$$

SOUTHWest Associated University, Kunming, China

\title{
TRANSFORMATIONS IN METRIC SPACES AND ORDINARY DIFFERENTIAL EQUATIONS
}

\author{
JOHN V. WEHAUSEN
}

1. Introduction. It is evident that the solutions of a differential equation $y^{\prime}=f(t, y)$ passing through a point $(\tau, \eta)$ in the region of definition of $f(t, y)$ may be considered as invariant functions of the transformation $T y(t)=\eta+\int_{\tau}^{t} f(s, y(s)) d s$ when suitable restrictions are placed upon the functions $y(t)$ considered. That such invariant functions exist for continuous $f(t, y)$ can be made a consequence of Schauder's fixed point theorem for completely continuous transformations in bounded convex subsets of a Banach space. ${ }^{1}$ For $f(t, y)$ satisfying a Lipschitz condition in $y$ the existence and uniqueness of an invariant function can be made to follow from a fixed point theorem of Caccioppoli of an essentially simpler nature. ${ }^{2}$ In the present paper we wish to show that the existence of invariant functions for continuous $f(t, y)$ as well as several other theorems concerning solutions of differential equations can be made to follow from some theorems concerning a particular class of transformations in a complete metric space. Although the existence theorem for fixed points given

Presented to the Society, November 26, 1943; received by the editors November 15, 1943, and, in revised form, June 20, 1944.

1 J. Schauder, Der Fixpunktsatz in Funktionalräumen, Studia Mathematica vol. 2 (1930) pp. 171-180; also, Zur Theorie stetiger Abbildungen in Funktionalräumen, Math. Zeit. vol. 26 (1927) pp. 47-65 and 417-431.

2 R. Caccioppoli, Un teorema generale sull'esistenza di elementi uniti in una trasformazione funzionale, Rendiconti R. Accademia dei Lincei (6) vol. 11 (1930) pp. 794799; for proofs and various applications of both Caccioppoli's and Schauder's theorems we refer also to Niemytsky, Metod nepodvizhnykh Tochek v Analize, Uspekhi Mat. Nauk vol. 1 (1936) pp. 141-174. 